\title{
THE COMPLEXITY OF INFINITE COMPUTATIONS IN MODELS OF SET THEORY
}

\author{
OLIVIER FINKEL
}

Equipe de Logique Mathématique, CNRS et Université Paris 7, France.

e-mail address: finkel@logique.jussieu.fr

\begin{abstract}
We prove the following surprising result: there exist a 1-counter Büchi automaton and a 2-tape Büchi automaton such that the $\omega$-language of the first and the infinitary rational relation of the second in one model of $\mathbf{Z F C}$ are $\mathbf{\Pi}_{2}^{0}$-sets, while in a different model of $\mathbf{Z F C}$ both are analytic but non Borel sets.

This shows that the topological complexity of an $\omega$-language accepted by a 1-counter Büchi automaton or of an infinitary rational relation accepted by a 2-tape Büchi automaton is not determined by the axiomatic system ZFC.

We show that a similar result holds for the class of languages of infinite pictures which are recognized by Büchi tiling systems.

We infer from the proof of the above results an improvement of the lower bound of some decision problems recently studied by the author.
\end{abstract}

\section{INTRODUCTION}

Acceptance of infinite words by finite automata was firstly considered in the sixties by Büchi in order to study the decidability of the monadic second order theory of one successor over the integers Büc62. The class of regular $\omega$-languages has been intensively studied and many applications have been found, see [Tho90, Sta97, PP04] for many results and references. Many extensions of regular $\omega$-languages have been investigated as the classes of $\omega$-languages accepted by 1-counter automata, pushdown automata, 2-tape automata, Petri nets, Turing machines, see [Tho90, EH93, Sta97, Fin08a] for a survey of this work.

A way to study the complexity of languages of infinite words accepted by finite machines is to study their topological complexity and firstly to locate them with regard to the Borel and the projective hierarchies. This work was analysed in Sta86, Sta87, Tho90, Sim92, EH93, LT94, Sta97]. It is well known that every $\omega$-language accepted by a deterministic Büchi automaton is a $\Pi_{2}^{0}$-set. This implies that any $\omega$-language accepted by a deterministic Muller automaton is a boolean combination of $\boldsymbol{\Pi}_{2}^{0}$-sets hence a $\boldsymbol{\Delta}_{3}^{0}$-set. But then it follows from Mc Naughton's Theorem, that all regular $\omega$-languages, which are accepted by deterministic Muller automata, are also $\boldsymbol{\Delta}_{3}^{0}$-sets. The Borel hierarchy of regular $\omega$-languages is

1998 ACM Subject Classification: F.1.1, F.1.3, F.4.1, F.4.3.

Key words and phrases: Infinite words; $\omega$-languages; 1-counter automaton; 2-tape automaton; twodimensional words; tiling systems; Cantor topology; topological complexity; Borel sets; largest effective coanalytic set; models of set theory; independence from the axiomatic system ZFC.

DOI:10.2168/LMCS-5 (4:4) 2009

(C) Olivier Finkel

(c) Creative Commons 
then determined. Moreover Landweber proved that one can effectively determine the Borel complexity of a regular $\omega$-language accepted by a given Muller or Büchi automaton, see [Lan69, Tho90, Sta97, PP04].

In recent papers [Fin06a, Fin08b] we have proved the following very surprising results. From the topological point of view, 1-counter Büchi automata and 2-tape Büchi automata have the same accepting power as Turing machines equipped with a Büchi acceptance condition. In particular, for every non null recursive ordinal $\alpha$, there exist some $\boldsymbol{\Sigma}_{\alpha}^{0}$-complete and some $\boldsymbol{\Pi}_{\alpha}^{0}$-complete 1-counter $\omega$-languages (respectively, infinitary rational relations). And the supremum of the set of Borel ranks of 1-counter $\omega$-languages (respectively, infinitary rational relations) is an ordinal $\gamma_{2}^{1}$ which is strictly greater than the first non recursive ordinal $\omega_{1}^{\mathrm{CK}}$. Moreover we have proved that there is no general algorithm to determine in an effective way the topological complexity of a given 1-counter $\omega$-language (respectively, infinitary rational relation). Topological properties of 1-counter $\omega$-languages (respectively, infinitary rational relations) are actually highly undecidable: for any countable ordinal $\alpha$, "determine whether a given 1-counter $\omega$-language (respectively, infinitary rational relation) is in the Borel class $\boldsymbol{\Sigma}_{\alpha}^{0}$ (respectively, $\boldsymbol{\Pi}_{\alpha}^{0}$ )" is a $\Pi_{2}^{1}$-hard problem, [Fin09b].

We prove here an even more amazing result which shows that Set Theory is actually very important in the study of infinite computations. Recall that the usual axiomatic system ZFC is Zermelo-Fraenkel system ZF plus the axiom of choice AC. We prove that there exist a 1-counter Büchi automaton $\mathcal{A}$ and a 2-tape Büchi automaton $\mathcal{B}$ such that :

(1) There is a model $V_{1}$ of $\mathbf{Z F C}$ in which the $\omega$-language $L(\mathcal{A})$ and the infinitary rational relation $L(\mathcal{B})$ are $\boldsymbol{\Pi}_{2}^{0}$-sets, and

(2) There is a model $V_{2}$ of $\mathbf{Z F C}$ in which the $\omega$-language $L(\mathcal{A})$ and the infinitary rational relation $L(\mathcal{B})$ are analytic but non Borel sets.

This shows that the topological complexity of an $\omega$-language accepted by a 1-counter Büchi automaton or of an infinitary rational relation accepted by a 2-tape Büchi automaton is not determined by the axiomatic system ZFC.

We show that a similar result holds for the class of languages of infinite pictures which are recognized by Büchi tiling systems, recently studied by Altenbernd, Thomas and Wöhrle in ATW03, see also [Fin04, Fin09a].

In order to prove these results, we consider the largest thin (i.e., without perfect subset) effective coanalytic subset of the Cantor space $2^{\omega}$. The existence of this largest thin $\Pi_{1^{-}}^{1}$ set $\mathcal{C}_{1}$ was proven by Kechris in Kec75 and independently by Guaspari and Sacks in Gua73, Sac76. By considering the cardinal of this set $\mathcal{C}_{1}$ in different models of set theory, we show that its topological complexity depends on the actual model of ZFC. Then we use some constructions from recent papers [Fin06a, Fin06b, Fin09a to infer our new results about 1-counter or 2-tape Büchi automata and Büchi tiling systems. From the proof of the above results and from Shoenfield's Absoluteness Theorem we get an improvement of the lower bound of some decision problems recently studied in Fin09b, Fin09a]. We show that the problem to determine whether an $\omega$-language accepted by a given real time 1-counter Büchi automaton (respectively, an infinitary rational relation accepted by a given 2-tape Büchi automaton) is in the Borel class $\boldsymbol{\Sigma}_{\alpha}^{0}$ (respectively, $\boldsymbol{\Pi}_{\alpha}^{0}$ ), for a countable ordinal $\alpha>2$ (respectively, $\alpha \geq 2$ ), is not in the class $\Pi_{2}^{1}$. A similar result holds for languages of infinite pictures accepted by Büchi tiling systems.

The paper is organized as follows. In Section 2 we recall definitions of counter automata, 2-tape automata, and tiling systems. We recall basic notions of topology in Section 3. 
Results on the largest effective coanalytic set are stated in Section 4. We prove our main results in Section 5.

Notice that as the results presented in this paper might be of interest to both set theorists and theoretical computer scientists, we shall recall in detail in Section 2 some notions of automata theory which are well known to computer scientists but not to set theorists. In a similar way we give in Sections 3 and 4 a presentation of some results of set theory which are well known to set theorists but not to computer scientists.

\section{Automata}

We assume now the reader to be familiar with the theory of formal $\omega$-languages Tho90, Sta97. We shall follow usual notations of formal language theory.

When $\Sigma$ is a finite alphabet, a non-empty finite word over $\Sigma$ is any sequence $x=$ $a_{1} \ldots a_{k}$, where $a_{i} \in \Sigma$ for $i=1, \ldots, k$, and $k$ is an integer $\geq 1$. The length of $x$ is $k$, denoted by $|x|$. The empty word has no letter and is denoted by $\lambda$; its length is $0 . \Sigma^{\star}$ is the set of finite words (including the empty word) over $\Sigma$.

The first infinite ordinal is $\omega$. An $\omega$-word over $\Sigma$ is an $\omega$-sequence $a_{1} \ldots a_{n} \ldots$, where for all integers $i \geq 1, a_{i} \in \Sigma$. When $\sigma$ is an $\omega$-word over $\Sigma$, we write $\sigma=\sigma(1) \sigma(2) \ldots \sigma(n) \ldots$, where for all $i, \sigma(i) \in \Sigma$, and $\sigma[n]=\sigma(1) \sigma(2) \ldots \sigma(n)$ for all $n \geq 1$ and $\sigma[0]=\lambda$.

The usual concatenation product of two finite words $u$ and $v$ is denoted $u . v$ (and sometimes just $u v$ ). This product is extended to the product of a finite word $u$ and an $\omega$-word $v$ : the infinite word $u . v$ is then the $\omega$-word such that:

$$
(u . v)(k)=u(k) \quad \text { if } k \leq|u| \text {, and } \quad(u . v)(k)=v(k-|u|) \quad \text { if } k>|u| .
$$

The set of $\omega$-words over the alphabet $\Sigma$ is denoted by $\Sigma^{\omega}$. An $\omega$-language over an alphabet $\Sigma$ is a subset of $\Sigma^{\omega}$. The complement (in $\Sigma^{\omega}$ ) of an $\omega$-language $V \subseteq \Sigma^{\omega}$ is $\Sigma^{\omega}-V$, denoted $V^{-}$.

For a finitary language $V \subseteq \Sigma^{\star}$, the $\omega$-power of $V$ is the $\omega$-language

$$
V^{\omega}=\left\{u_{1} \ldots u_{n} \ldots \in \Sigma^{\omega} \mid \forall i \geq 1 u_{i} \in V\right\}
$$

Abstract models of finite machines reading finite or infinite words have been considered in automata theory, calculability and complexity theories. The simplest model of machine used for recognizability of languages of (finite or infinite) words is the model of finite state machine. One can consider that such a machine $\mathcal{M}$ has a semi infinite tape divided into cells. This tape contains at the beginning the input word written from left to right, each letter being contained in a cell; in the case of a finite input word, the remaining cells contain a special blank symbol. The machine has a reading (only) head, placed at the beginning on the first cell. It has also a finite control, consisting of a finite set $K$ of states and a current state. There is a special state $q_{0}$ called the initial state and a set $F \subseteq K$ of final states. The reading of a word begins in state $q_{0}$; then the machine reads successively the letters from left to right, changing the current state according to the transition relation which has a finite description. The finite word $x$ is accepted by $\mathcal{M}$ if the reading of $x$ ends in a final state. An infinite word $\sigma$ is accepted by $\mathcal{M}$ if some final state occurs infinitely often during the reading of $\sigma$. We now give a formal definition of a finite state machine.

Definition 2.1. A finite state machine (FSM) is a quadruple $\mathcal{M}=\left(K, \Sigma, \delta, q_{0}\right)$, where $K$ is a finite set of states, $\Sigma$ is a finite input alphabet, $q_{0} \in K$ is the initial state and $\delta$ is a mapping from $K \times \Sigma$ into $2^{K}$. 
Let $x=a_{1} a_{2} \ldots a_{n}$ be a finite word over $\Sigma$. A sequence of states $r=q_{1} q_{2} \ldots q_{n} q_{n+1}$ is called a run of $\mathcal{M}$ on $x$ iff:

(1) $q_{1}=q_{0}$ is the initial state, and

(2) for each $i \geq 1, q_{i+1} \in \delta\left(q_{i}, a_{i}\right)$.

Let $\sigma=a_{1} a_{2} \ldots a_{n} \ldots$ be an $\omega$-word over $\Sigma$. A sequence of states $r=q_{1} q_{2} \ldots q_{n} \ldots$ is called an (infinite) run of $\mathcal{M}$ on $\sigma$ iff:

(1) $q_{1}=q_{0}$ is the initial state, and

(2) for each $i \geq 1, q_{i+1} \in \delta\left(q_{i}, a_{i}\right)$.

For every (infinite) run $r=q_{1} q_{2} \ldots q_{n} \ldots$ of $\mathcal{M}, \operatorname{In}(r)$ is the set of states entered infinitely often by $\mathcal{M}$ during the run $r$.

Definition 2.2. An automaton is a 5 -tuple $\mathcal{M}=\left(K, \Sigma, \delta, q_{0}, F\right)$ where $\mathcal{M}^{\prime}=\left(K, \Sigma, \delta, q_{0}\right)$ is a finite state machine and $F \subseteq K$ is the set of final states. The language accepted by $\mathcal{M}$ is the set of finite words $x$ such that there is a run of $\mathcal{M}$ on $x$ ending in a final state.

Definition 2.3. A Büchi automaton is a 5-tuple $\mathcal{M}=\left(K, \Sigma, \delta, q_{0}, F\right)$ where $\mathcal{M}^{\prime}=\left(K, \Sigma, \delta, q_{0}\right)$ is a finite state machine and $F \subseteq K$ is the set of final states. The $\omega$-language accepted by $\mathcal{M}$ is

$$
L(\mathcal{M})=\left\{\sigma \in \Sigma^{\omega} \mid \text { there exists a run } r \text { of } \mathcal{M} \text { on } \sigma \text { such that } \operatorname{In}(r) \cap F \neq \emptyset\right\} .
$$

Recall that a language (respectively, $\omega$-language) is said to be regular iff it is accepted by an automaton (respectively, Büchi automaton). An $\omega$-language $L$ is regular iff it belongs to the $\omega$-Kleene closure of the class of finitary regular languages, i.e. iff there exist some regular languages $U_{i}, V_{i}$, for $i \in[1, n]$, such that $L=\bigcup_{i=1}^{n} U_{i} . V_{i}^{\omega}$.

Notice that a finite state machine has only a bounded memory containing the current state of the machine. More complicated machines have been considered which can store some unbounded contents. In particular a counter machine has a finite set of counters, each of which containing a non-negative integer. The machine can test whether the content of a given counter is zero or not. And transitions depend on the letter read by the machine, the current state of the finite control, and the tests about the values of the counters. Each transition leads to another state, and values of the counters can be increased by 1 or decreased by 1 , providing that these values always remain non-negatives. Notice that in this model some $\lambda$-transitions are allowed. During these transitions the reading head of the machine does not move to the right, i.e. the machine does not read any more letter.

We now recall the formal definition of $k$-counter machine and $k$-counter Büchi automata which will be useful in the sequel.

Definition 2.4. Let $k$ be an integer $\geq 1$. A $k$-counter machine is a 4 -tuple $\mathcal{M}=(K, \Sigma$, $\left.\Delta, q_{0}\right)$, where $K$ is a finite set of states, $\Sigma$ is a finite input alphabet, $q_{0} \in K$ is the initial state, and $\Delta \subseteq K \times(\Sigma \cup\{\lambda\}) \times\{0,1\}^{k} \times K \times\{0,1,-1\}^{k}$ is the transition relation. The $k$-counter machine $\mathcal{M}$ is said to be real time iff: $\Delta \subseteq K \times \Sigma \times\{0,1\}^{k} \times K \times\{0,1,-1\}^{k}$, i.e. iff there are no $\lambda$-transitions.

If the machine $\mathcal{M}$ is in state $q$ and $c_{i} \in \mathbb{N}$ is the content of the $i^{\text {th }}$ counter $\mathcal{C}_{i}$ then the configuration (or global state) of $\mathcal{M}$ is the $(k+1)$-tuple $\left(q, c_{1}, \ldots, c_{k}\right)$.

For $a \in \Sigma \cup\{\lambda\}, q, q^{\prime} \in K$ and $\left(c_{1}, \ldots, c_{k}\right) \in \mathbb{N}^{k}$ such that $c_{j}=0$ for $j \in E \subseteq\{1, \ldots, k\}$ and $c_{j}>0$ for $j \notin E$, if $\left(q, a, i_{1}, \ldots, i_{k}, q^{\prime}, j_{1}, \ldots, j_{k}\right) \in \Delta$ where $i_{j}=0$ for $j \in E$ and $i_{j}=1$ for $j \notin E$, then we write:

$$
a:\left(q, c_{1}, \ldots, c_{k}\right) \mapsto \mathcal{M}\left(q^{\prime}, c_{1}+j_{1}, \ldots, c_{k}+j_{k}\right)
$$


Thus we see that the transition relation must satisfy:

- if $\left(q, a, i_{1}, \ldots, i_{k}, q^{\prime}, j_{1}, \ldots, j_{k}\right) \in \Delta$ and $i_{m}=0$ for some $m \in\{1, \ldots, k\}$, then $j_{m}=0$ or $j_{m}=1$ (but $j_{m}$ may not be equal to -1 ).

Let $\sigma=a_{1} a_{2} \ldots a_{n} \ldots$ be an $\omega$-word over $\Sigma$. An $\omega$-sequence of configurations $r=\left(q_{i}, c_{1}^{i}, \ldots c_{k}^{i}\right)_{i \geq 1}$ is called a run of $\mathcal{M}$ on $\sigma$, starting in configuration $\left(p, c_{1}, \ldots, c_{k}\right)$, iff:

(1) $\left(q_{1}, c_{1}^{1}, \ldots c_{k}^{1}\right)=\left(p, c_{1}, \ldots, c_{k}\right)$

(2) for each $i \geq 1$, there exists $b_{i} \in \Sigma \cup\{\lambda\}$ such that $b_{i}:\left(q_{i}, c_{1}^{i}, \ldots c_{k}^{i}\right) \mapsto \mathcal{M}\left(q_{i+1}, c_{1}^{i+1}, \ldots c_{k}^{i+1}\right)$ and such that either $a_{1} a_{2} \ldots a_{n} \ldots=b_{1} b_{2} \ldots b_{n} \ldots$ or $b_{1} b_{2} \ldots b_{n} \ldots$ is a finite prefix of $a_{1} a_{2} \ldots a_{n} \ldots$

The run $r$ is said to be complete when $a_{1} a_{2} \ldots a_{n} \ldots=b_{1} b_{2} \ldots b_{n} \ldots$

For every such run, $\operatorname{In}(r)$ is the set of all states entered infinitely often during the run $r$.

A complete run $r$ of $M$ on $\sigma$, starting in configuration $\left(q_{0}, 0, \ldots, 0\right)$, will be simply called "a run of $M$ on $\sigma$ ".

Definition 2.5. A Büchi $k$-counter automaton is a 5-tuple $\mathcal{M}=\left(K, \Sigma, \Delta, q_{0}, F\right)$, where $\mathcal{M}^{\prime}=\left(K, \Sigma, \Delta, q_{0}\right)$ is a $k$-counter machine and $F \subseteq K$ is the set of accepting states. The $\omega$ language accepted by $\mathcal{M}$ is $L(\mathcal{M})=\left\{\sigma \in \Sigma^{\omega} \mid\right.$ there exists a run $\mathrm{r}$ of $\mathcal{M}$ on $\sigma$ such that $\operatorname{In}(r) \cap$ $F \neq \emptyset\}$.

The class of $\omega$-languages accepted by Büchi $k$-counter automata will be denoted $\mathbf{B C L}(k)_{\omega}$. The class of $\omega$-languages accepted by real time Büchi $k$-counter automata will be denoted $\mathbf{r}-\mathbf{B C L}(k)_{\omega}$.

Remark that the 1-counter automata introduced above are equivalent to the pushdown automata whose stack alphabet is in the form $\left\{Z_{0}, A\right\}$ where $Z_{0}$ is the bottom symbol which always remains at the bottom of the stack and appears only there and $A$ is another stack symbol, see ABB96.

The class BCL $(1)_{\omega}$ is a strict subclass of the class $\mathbf{C F L}_{\omega}$ of context free $\omega$-languages accepted by Büchi pushdown automata. Notice that an $\omega$-language $L$ is in the class $\mathbf{B C L}(1)_{\omega}$ (respectively, $\mathbf{C F L}_{\omega}$ ) iff it belongs to the $\omega$-Kleene closure of the class of finitary languages accepted by 1-counter automata (respectively, pushdown automata), i.e. iff there exist some 1-counter (respectively, context-free) languages $U_{i}, V_{i}$, for $i \in[1, n]$, such that $L=\bigcup_{i=1}^{n} U_{i} \cdot V_{i}^{\omega}$, see [Sta97, Fin06a, Fin08a].

We shall consider also the notion of acceptance of binary relations over infinite words by 2-tape Büchi automata, firstly considered by Gire and Nivat in Gir81, GN84. A 2-tape automaton is an automaton having two tapes and two reading heads, one for each tape, which can move asynchronously, and a finite control as in the case of a (1-tape) automaton. The automaton reads a pair of (infinite) words $(u, v)$ where $u$ is on the first tape and $v$ is on the second tape. Such automata can also be considered for the reading of pairs of finite words but we shall only need here the case of infinite words. We now recall the formal definition of 2-tape Büchi automata and of infinitary rational relations.

Definition 2.6. A 2-tape Büchi automaton is a 6-tuple $\mathcal{T}=\left(K, \Sigma_{1}, \Sigma_{2}, \Delta, q_{0}, F\right)$, where $K$ is a finite set of states, $\Sigma_{1}$ and $\Sigma_{2}$ are finite alphabets, $\Delta$ is a finite subset of $K \times \Sigma_{1}^{\star} \times \Sigma_{2}^{\star} \times K$ called the set of transitions, $q_{0}$ is the initial state, and $F \subseteq K$ is the set of accepting states.

A computation $\mathcal{C}$ of the 2-tape Büchi automaton $\mathcal{T}$ is an infinite sequence of transitions

$$
\left(q_{0}, u_{1}, v_{1}, q_{1}\right),\left(q_{1}, u_{2}, v_{2}, q_{2}\right), \ldots\left(q_{i-1}, u_{i}, v_{i}, q_{i}\right),\left(q_{i}, u_{i+1}, v_{i+1}, q_{i+1}\right), \ldots
$$


The computation is said to be successful iff there exists a final state $q_{f} \in F$ and infinitely many integers $i \geq 0$ such that $q_{i}=q_{f}$. The input word of the computation is $u=u_{1} \cdot u_{2} . u_{3} \ldots$ The output word of the computation is $v=v_{1} \cdot v_{2} \cdot v_{3} \ldots$ Here the input and the output words may be finite or infinite.

The infinitary rational relation $L(\mathcal{T}) \subseteq \Sigma_{1}^{\omega} \times \Sigma_{2}^{\omega}$ accepted by the 2-tape Büchi automaton $\mathcal{T}$ is the set of pairs $(u, v) \in \Sigma_{1}^{\omega} \times \Sigma_{2}^{\omega}$ such that $u$ and $v$ are the input and the output words of some successful computation $\mathcal{C}$ of $\mathcal{T}$.

Remark 2.7. An infinitary rational relation $L(\mathcal{T}) \subseteq \Sigma_{1}^{\omega} \times \Sigma_{2}^{\omega}$ may be seen as an $\omega$-language over the product alphabet $\Sigma_{1} \times \Sigma_{2}$.

In the sequel, we will also consider the notion of recognizable language of infinite pictures. We recall first some basic definitions about languages of infinite two-dimensional words, i.e., languages of infinite pictures.

Let $\Sigma$ be a finite alphabet and \# be a letter not in $\Sigma$ and let $\hat{\Sigma}=\Sigma \cup\{\#\}$. An $\omega$-picture over $\Sigma$ is a function $p$ from $\omega \times \omega$ into $\hat{\Sigma}$ such that $p(i, 0)=p(0, i)=\#$ for all $i \geq 0$ and $p(i, j) \in \Sigma$ for $i, j>0$. For each integer $j \geq 1$, the $j^{\text {th }}$ row of the $\omega$-picture $p$ is the infinite word $p(1, j) \cdot p(2, j) \cdot p(3, j) \ldots$ over $\Sigma$ and the $j^{\text {th }}$ column of $p$ is the infinite word $p(j, 1) \cdot p(j, 2) \cdot p(j, 3) \ldots$ over $\Sigma$. The set of $\omega$-pictures over $\Sigma$ is denoted by $\Sigma^{\omega, \omega}$. An $\omega$-picture language $L$ is a subset of $\Sigma^{\omega, \omega}$.

In ATW03, Altenbernd, Thomas and Wöhrle have considered acceptance of languages of infinite two-dimensional words (infinite pictures) by finite tiling systems, with the usual acceptance conditions, such as the Büchi and Muller ones, firstly used for infinite words. They showed that Büchi and Muller acceptance conditions lead to the same class of recognizable languages of infinite pictures. So we shall only recall the notion of Büchi recognizable languages of infinite pictures, see [ATW03, Fin04, Fin09a for more details.

A tiling system is a tuple $\mathcal{A}=(Q, \Sigma, \Delta)$, where $Q$ is a finite set of states, $\Sigma$ is a finite alphabet, $\Delta \subseteq(\hat{\Sigma} \times Q)^{4}$ is a finite set of tiles.

A Büchi tiling system is a pair $(\mathcal{A}, F)$ where $\mathcal{A}=(Q, \Sigma, \Delta)$ is a tiling system and $F \subseteq Q$ is the set of accepting states. Tiles are denoted by

$$
\left(\begin{array}{ll}
\left(a_{3}, q_{3}\right) & \left(a_{4}, q_{4}\right) \\
\left(a_{1}, q_{1}\right) & \left(a_{2}, q_{2}\right)
\end{array}\right)
$$

with $a_{i} \in \hat{\Sigma}$ and $q_{i} \in Q$, and in general, over an alphabet $\Gamma$, by

$$
\left(\begin{array}{ll}
b_{3} & b_{4} \\
b_{1} & b_{2}
\end{array}\right)
$$

with $b_{i} \in \Gamma$. A combination of tiles is defined by:

$$
\left(\begin{array}{ll}
b_{3} & b_{4} \\
b_{1} & b_{2}
\end{array}\right) \circ\left(\begin{array}{ll}
b_{3}^{\prime} & b_{4}^{\prime} \\
b_{1}^{\prime} & b_{2}^{\prime}
\end{array}\right)=\left(\begin{array}{cc}
\left(b_{3}, b_{3}^{\prime}\right) & \left(b_{4}, b_{4}^{\prime}\right) \\
\left(b_{1}, b_{1}^{\prime}\right) & \left(b_{2}, b_{2}^{\prime}\right)
\end{array}\right)
$$

Definition 2.8. Let $\mathcal{A}=(Q, \Sigma, \Delta)$ be a tiling system, and $F \subseteq Q$ be the set of accepting states.

A run of the tiling system $\mathcal{A}=(Q, \Sigma, \Delta)$ over an $\omega$-picture $p \in \Sigma^{\omega, \omega}$ is a mapping $\rho$ from $\omega \times \omega$ into $Q$ such that for all $(i, j) \in \omega \times \omega$ with $p(i, j)=a_{i, j}$ and $\rho(i, j)=q_{i, j}$ we have

$$
\left(\begin{array}{cc}
a_{i, j+1} & a_{i+1, j+1} \\
a_{i, j} & a_{i+1, j}
\end{array}\right) \circ\left(\begin{array}{cc}
q_{i, j+1} & q_{i+1, j+1} \\
q_{i, j} & q_{i+1, j}
\end{array}\right) \in \Delta .
$$


The $\omega$-picture language $L((\mathcal{A}, F))$ Büchi-recognized by $(\mathcal{A}, F)$ is the set of $\omega$-pictures $p \in \Sigma^{\omega, \omega}$ such that there is some run $\rho$ of $\mathcal{A}$ on $p$ and $\rho(v) \in F$ for infinitely many $v \in \omega^{2}$.

An interesting variation of the above defined reognizability condition for infinite pictures uses the diagonal of an $\omega$-picture. The diagonal of an $\omega$-picture $p$ is the set of vertices $D i(p)=\{(i, i) \mid i \in \omega\}$.

The $\omega$-picture language Büchi-recognized by $(\mathcal{A}, F)$ on the diagonal is the set of $\omega$ pictures $p \in \Sigma^{\omega, \omega}$ such that there is some run $\rho$ of $\mathcal{A}$ on $p$ and $\rho(v) \in F$ for infinitely many $v \in \operatorname{Di}(p)$.

The following result was stated in ATW03.

Theorem 2.9. An $\omega$-picture language $L \subseteq \Sigma^{\omega, \omega}$ is Büchi-recognized by a tiling system if and only if it is Büchi-recognized on the diagonal by a tiling system.

We can state some links with classical notions of tiling of the (quarter of the) plane, see for instance [BJ08].

We denote $\Gamma=\hat{\Sigma} \times Q$ where $\Sigma$ is the alphabet of pictures and $Q$ is the set of states of a tiling system $\mathcal{A}=(Q, \Sigma, \Delta)$. We consider configurations which are elements of $\Gamma^{\omega \times \omega}$. One can imagine that each cell of the quarter of the plane contains a letter of the alphabet $\Gamma$.

Let $\Delta \subseteq(\hat{\Sigma} \times Q)^{4}=\Gamma^{4}$ be a finite set of tiles. We denote its complement by $\Delta^{-}=$ $\Gamma^{4}-\Delta$. A tiling of the (quarter of the) plane with $\Delta^{-}$as set of forbidden patterns is simply a configuration $c \in \Gamma^{\omega \times \omega}$ such that for all integers $i, j \in \omega$ :

$$
\left(\begin{array}{cc}
c(i, j+1) & c(i+1, j+1) \\
c(i, j) & c(i+1, j)
\end{array}\right) \in \Delta .
$$

Then the $\omega$-picture language $L \subseteq \Sigma^{\omega, \omega}$ which is Büchi-recognized on the diagonal by the tiling system $(\mathcal{A}, F)$ is simply the set of $\omega$-pictures $p \in \Sigma^{\omega, \omega}$ which are projections of configurations $c \in \Gamma^{\omega \times \omega}$ which are tilings of the (quarter of the) plane with $\Delta^{-}$as set of forbidden patterns such that for infinitely many $i \in \omega$ the second component of $c(i, i)$ is in $F$.

\section{TOPOLOGY}

We assume the reader to be familiar with basic notions of topology which may be found in [Mos80, LT94, Kec95, Sta97, PP04]. There is a natural metric on the set $\Sigma^{\omega}$ of infinite words over a finite alphabet $\Sigma$ containing at least two letters which is called the prefix metric and defined as follows. For $u, v \in \Sigma^{\omega}$ and $u \neq v$ let $\delta(u, v)=2^{-l_{\operatorname{pref}(u, v)} \text { where } l_{\operatorname{pref}(u, v)} \text { is }}$ the first integer $n$ such that the $(n+1)^{s t}$ letter of $u$ is different from the $(n+1)^{s t}$ letter of $v$. This metric induces on $\Sigma^{\omega}$ the usual Cantor topology for which open subsets of $\Sigma^{\omega}$ are in the form $W . \Sigma^{\omega}$, where $W \subseteq \Sigma^{\star}$. A set $L \subseteq \Sigma^{\omega}$ is a closed set iff its complement $\Sigma^{\omega}-L$ is an open set. Define now the Borel Hierarchy of subsets of $\Sigma^{\omega}$ :

Definition 3.1. For a non-null countable ordinal $\alpha$, the classes $\boldsymbol{\Sigma}_{\alpha}^{0}$ and $\boldsymbol{\Pi}_{\alpha}^{0}$ of the Borel Hierarchy on the topological space $\Sigma^{\omega}$ are defined as follows:

$-\boldsymbol{\Sigma}_{1}^{0}$ is the class of open subsets of $\Sigma^{\omega}$,

$-\Pi_{1}^{0}$ is the class of closed subsets of $\Sigma^{\omega}$, and for any countable ordinal $\alpha \geq 2$ :

- $\boldsymbol{\Sigma}_{\alpha}^{0}$ is the class of countable unions of subsets of $\Sigma^{\omega}$ in $\bigcup_{\gamma<\alpha} \Pi_{\gamma}^{0}$.

$-\boldsymbol{\Pi}_{\alpha}^{0}$ is the class of countable intersections of subsets of $\Sigma^{\omega}$ in $\bigcup_{\gamma<\alpha} \boldsymbol{\Sigma}_{\gamma}^{0}$. 
Recall some basic results about these classes. The Borel classes are closed under finite intersections and unions, and continuous preimages. Moreover, $\boldsymbol{\Sigma}_{\xi}^{0}$ is closed under countable unions, and $\boldsymbol{\Pi}_{\xi}^{0}$ under countable intersections. As usual the ambiguous class $\boldsymbol{\Delta}_{\xi}^{0}$ is the class $\Sigma_{\xi}^{0} \cap \Pi_{\xi}^{0}$.

The class of Borel sets is $\boldsymbol{\Delta}_{1}^{1}:=\bigcup_{\xi<\omega_{1}} \boldsymbol{\Sigma}_{\xi}^{0}=\bigcup_{\xi<\omega_{1}} \boldsymbol{\Pi}_{\xi}^{0}$, where $\omega_{1}$ is the first uncountable ordinal. The class of Borel sets is the closure of the class of open sets under countable union and countable intersection. It is also the closure of the class of open sets under countable union (respectively, intersection) and complementation.

The Borel hierarchy is as follows:

$$
\begin{array}{lllllll} 
& \boldsymbol{\Sigma}_{1}^{0}=\text { open } & & \boldsymbol{\Sigma}_{2}^{0} & \ldots & \boldsymbol{\Sigma}_{\omega}^{0} & \ldots \\
& \boldsymbol{\Delta}_{2}^{0} & & & \boldsymbol{\Delta}_{\omega}^{0} & & \boldsymbol{\Delta}_{1}^{1} \\
& \boldsymbol{\Pi}_{1}^{0}=\text { cloped } & & \boldsymbol{\Pi}_{2}^{0} & \ldots & \boldsymbol{\Pi}_{\omega}^{0} & \ldots
\end{array}
$$

This picture means that any class is contained in every class to the right of it, and the inclusion is strict in any of the spaces $\Sigma^{\omega}$.

For a countable ordinal $\alpha$, a subset of $\Sigma^{\omega}$ is a Borel set of rank $\alpha$ iff it is in $\boldsymbol{\Sigma}_{\alpha}^{0} \cup \boldsymbol{\Pi}_{\alpha}^{0}$ but not in $\bigcup_{\gamma<\alpha}\left(\boldsymbol{\Sigma}_{\gamma}^{0} \cup \boldsymbol{\Pi}_{\gamma}^{0}\right)$.

There are also some subsets of $\Sigma^{\omega}$ which are not Borel. Indeed there exists another hierarchy beyond the Borel hierarchy, which is called the projective hierarchy and which is obtained from the Borel hierarchy by successive applications of operations of projection and complementation. The first level of the projective hierarchy is formed by the class of analytic sets and the class of co-analytic sets which are complements of analytic sets. In particular the class of Borel subsets of $\Sigma^{\omega}$ is strictly included into the class $\boldsymbol{\Sigma}_{1}^{1}$ of analytic sets which are obtained by projection of Borel sets.

Definition 3.2. A subset $A$ of $\Sigma^{\omega}$ is in the class $\Sigma_{1}^{1}$ of analytic sets iff there exists another finite set $Y$ and a Borel subset $B$ of $(\Sigma \times Y)^{\omega}$ such that $x \in A \leftrightarrow \exists y \in Y^{\omega}$ such that $(x, y) \in$ $B$, where $(x, y)$ is the infinite word over the alphabet $\Sigma \times Y$ such that $(x, y)(i)=(x(i), y(i))$ for each integer $i \geq 1$.

Remark 3.3. In the above definition we could take $B$ in the class $\boldsymbol{\Pi}_{2}^{0}$. Moreover analytic subsets of $\Sigma^{\omega}$ are the projections of $\Pi_{1}^{0}$-subsets of $\Sigma^{\omega} \times \omega^{\omega}$, where $\omega^{\omega}$ is the Baire space, Mos80.

By Suslin's Theorem it holds that a subset $A$ of $\Sigma^{\omega}$ is Borel iff it is analytic and coanalytic, i.e. $\boldsymbol{\Delta}_{1}^{1}=\boldsymbol{\Pi}_{1}^{1} \cap \boldsymbol{\Sigma}_{1}^{1}$. A set $A$ which is analytic but not coanalytic, or equivalently analytic but not Borel, is called a true analytic set.

We now define completeness with regard to reduction by continuous functions. For a countable ordinal $\alpha \geq 1$, a set $F \subseteq \Sigma^{\omega}$ is said to be a $\boldsymbol{\Sigma}_{\alpha}^{0}$ (respectively, $\boldsymbol{\Pi}_{\alpha}^{0}, \boldsymbol{\Sigma}_{1}^{1}$ )-complete set iff for any set $E \subseteq Y^{\omega}$ (with $Y$ a finite alphabet): $E \in \Sigma_{\alpha}^{0}$ (respectively, $E \in \boldsymbol{\Pi}_{\alpha}^{0}$, $\left.E \in \Sigma_{1}^{1}\right)$ iff there exists a continuous function $f: Y^{\omega} \rightarrow \Sigma^{\omega}$ such that $E=f^{-1}(F)$.

Recall that a set $X \subseteq \Sigma^{\omega}$ is a $\boldsymbol{\Sigma}_{\alpha}^{0}$ (respectively $\boldsymbol{\Pi}_{\alpha}^{0}$ )-complete subset of $\Sigma^{\omega}$ iff it is in $\boldsymbol{\Sigma}_{\alpha}^{0}$ but not in $\boldsymbol{\Pi}_{\alpha}^{\mathbf{0}}$ (respectively in $\boldsymbol{\Pi}_{\alpha}^{0}$ but not in $\boldsymbol{\Sigma}_{\alpha}^{0}$ ), Kec95. $\boldsymbol{\Sigma}_{n}^{0}$ (respectively $\boldsymbol{\Pi}_{n}^{0}$ )-complete sets, with $n$ an integer $\geq 1$, are thoroughly characterized in [Sta86].

In particular, the singletons of $2^{\omega}$ are $\Pi_{1}^{0}$-complete subsets of $2^{\omega}$. The $\omega$-language $\mathcal{R}=\left(0^{\star} .1\right)^{\omega}$ is a well known example of $\boldsymbol{\Pi}_{2}^{0}$-complete subset of $\{0,1\}^{\omega}$. It is the set of $\omega$-words over $\{0,1\}$ having infinitely many occurrences of the letter 1 . Its complement $\{0,1\}^{\omega}-\left(0^{\star} .1\right)^{\omega}$ is a $\boldsymbol{\Sigma}_{2}^{0}$-complete subset of $\{0,1\}^{\omega}$. 
We recall now the definition of the arithmetical hierarchy of $\omega$-languages which form the effective analogue to the hierarchy of Borel sets of finite ranks.

Let $X$ be a finite alphabet. An $\omega$-language $L \subseteq X^{\omega}$ belongs to the class $\Sigma_{n}$ if and only if there exists a recursive relation $R_{L} \subseteq(\mathbb{N})^{n-1} \times X^{\star}$ such that

$$
L=\left\{\sigma \in X^{\omega} \mid \exists a_{1} \ldots Q_{n} a_{n} \quad\left(a_{1}, \ldots, a_{n-1}, \sigma\left[a_{n}+1\right]\right) \in R_{L}\right\}
$$

where $Q_{i}$ is one of the quantifiers $\forall$ or $\exists$ (not necessarily in an alternating order). An $\omega$-language $L \subseteq X^{\omega}$ belongs to the class $\Pi_{n}$ if and only if its complement $X^{\omega}-L$ belongs to the class $\Sigma_{n}$. The inclusion relations that hold between the classes $\Sigma_{n}$ and $\Pi_{n}$ are the same as for the corresponding classes of the Borel hierarchy. The classes $\Sigma_{n}$ and $\Pi_{n}$ are included in the respective classes $\boldsymbol{\Sigma}_{\mathbf{n}}^{\mathbf{0}}$ and $\boldsymbol{\Sigma}_{\mathbf{n}}^{\mathbf{0}}$ of the Borel hierarchy, and cardinality arguments suffice to show that these inclusions are strict.

As in the case of the Borel hierarchy, projections of arithmetical sets (of the second $\Pi$-class) lead beyond the arithmetical hierarchy, to the analytical hierarchy of $\omega$-languages. The first class of this hierarchy is the (lightface) class $\Sigma_{1}^{1}$ of effective analytic sets which are obtained by projection of arithmetical sets. An $\omega$-language $L \subseteq X^{\omega}$ belongs to the class $\Sigma_{1}^{1}$ if and only if there exists a recursive relation $R_{L} \subseteq \mathbb{N} \times\{0,1\}^{\star} \times X^{\star}$ such that:

$$
L=\left\{\sigma \in X^{\omega} \mid \exists \tau\left(\tau \in\{0,1\}^{\omega} \wedge \forall n \exists m\left((n, \tau[m], \sigma[m]) \in R_{L}\right)\right)\right\}
$$

Then an $\omega$-language $L \subseteq X^{\omega}$ is in the class $\Sigma_{1}^{1}$ iff it is the projection of an $\omega$ language over the alphabet $X \times\{0,1\}$ which is in the class $\Pi_{2}$. The (lightface) class $\Pi_{1}^{1}$ of effective co-analytic sets is simply the class of complements of effective analytic sets. We denote as usual $\Delta_{1}^{1}=\Sigma_{1}^{1} \cap \Pi_{1}^{1}$.

Recall that an $\omega$-language $L \subseteq X^{\omega}$ is in the class $\Sigma_{1}^{1}$ iff it is accepted by a non deterministic Turing machine (reading $\omega$-words) with a Büchi or Muller acceptance condition CG78, Sta97.

\section{The LARgest thin EFFECTIVE COANALYTiC SET}

We now recall some basic notions of set theory which will be useful in the sequel, and which are exposed in any textbook on set theory, such as [Jec02].

The usual axiomatic system ZFC is Zermelo-Fraenkel system ZF plus the axiom of choice AC. A model $(\mathbf{V}, \in)$ of the axiomatic system $\mathbf{Z F C}$ is a collection $\mathbf{V}$ of sets, equipped with the membership relation $\in$, where " $x \in y$ " means that the set $x$ is an element of the set $y$, which satisfies the axioms of ZFC. We shall often say " the model V" instead of "the model $(\mathbf{V}, \in)$ ".

The axioms of ZFC express some natural facts that we consider to hold in the universe of sets. For instance a natural fact is that two sets $x$ and $y$ are equal iff they have the same elements. This is expressed by the sentence:

$$
\forall x \forall y[x=y \leftrightarrow \forall z(z \in x \leftrightarrow z \in y)]
$$

The above sentence is the Axiom of Extensionality.

Another natural axiom is the Pairing Axiom which states that for all sets $x$ and $y$ there exists a set $z=\{x, y\}$ whose elements are $x$ and $y$ :

$$
\forall x \forall y[\exists z(\forall w(w \in z \leftrightarrow(w=x \vee w=y)))]
$$

Similarly the Powerset Axiom states the existence of the set of subsets of a set $x$. 
The Separation Schema is in fact an infinite set of axioms. For each first-order formula $\varphi$, with free variable $z$, in the language of set theory with the equality symbol and the binary symbol $\in$, the following axiom states the existence of the set $y=\{z \in x \mid \varphi(z)\}$ of elements of a set $x$ which satisfy $\varphi$.

$$
\forall x[\exists y(\forall z(z \in y \leftrightarrow(z \in x \wedge \varphi(z))))]
$$

The other axioms of ZFC are the Union Axiom, the Replacement Schema, the Infinity Axiom, the Foundation Axiom, and the Axiom of Choice. We refer the reader to any textbook on set theory, like [Jec02], for an exposition of these axioms.

We recall that the infinite cardinals are usually denoted by $\aleph_{0}, \aleph_{1}, \aleph_{2}, \ldots, \aleph_{\alpha}, \ldots$ The cardinal $\aleph_{\alpha}$ is also denoted by $\omega_{\alpha}$, as usual when it is considered as an ordinal.

The continuum hypothesis $\mathbf{C H}$ says that the first uncountable cardinal $\aleph_{1}$ is equal to $2^{\aleph_{0}}$ which is the cardinal of the continuum. Gödel and Cohen have proved that the continuum hypothesis $\mathbf{C H}$ is independent from the axiomatic system ZFC. This means that there are some models of $\mathbf{Z F C}+\mathbf{C H}$ and also some models of $\mathbf{Z F C}+\neg \mathbf{C H}$, where $\neg \mathbf{C H}$ denotes the negation of the continuum hypothesis, Jec02.

Let $\mathbf{O N}$ be the class of all ordinals. Recall that an ordinal $\alpha$ is said to be a successor ordinal iff there exists an ordinal $\beta$ such that $\alpha=\beta+1$; otherwise the ordinal $\alpha$ is said to be a limit ordinal and in that case $\alpha=\sup \{\beta \in \mathbf{O N} \mid \beta<\alpha\}$.

The class $\mathbf{L}$ of constructible sets in a model $\mathbf{V}$ of $\mathbf{Z F}$ is defined by

$$
\mathbf{L}=\bigcup_{\alpha \in \mathbf{O N}} \mathbf{L}(\alpha)
$$

where the sets $\mathbf{L}(\alpha)$ are constructed by induction as follows:

(1) $\mathbf{L}(0)=\emptyset$

(2) $\mathbf{L}(\alpha)=\bigcup_{\beta<\alpha} \mathbf{L}(\beta)$, for $\alpha$ a limit ordinal, and

(3) $\mathbf{L}(\alpha+1)$ is the set of subsets of $\mathbf{L}(\alpha)$ which are definable from a finite number of elements of $\mathbf{L}(\alpha)$ by a first-order formula relativized to $\mathbf{L}(\alpha)$.

If $\mathbf{V}$ is a model of $\mathbf{Z F}$ and $\mathbf{L}$ is the class of constructible sets of $\mathbf{V}$, then the class $\mathbf{L}$ forms a model of $\mathbf{Z F C}+\mathbf{C H}$. Notice that the axiom $(\mathbf{V}=\mathbf{L})$ means "every set is constructible" and that it is consistent with $\mathbf{Z F C}$.

Consider now a model $\mathbf{V}$ of the axiomatic system ZFC and the class of constructible sets $\mathbf{L} \subseteq \mathbf{V}$ which forms another model of $\mathbf{Z F C}$. It is known that the ordinals of $\mathbf{L}$ are also the ordinals of $\mathbf{V}$. But the cardinals in $\mathbf{V}$ may be different from the cardinals in $\mathbf{L}$.

In the sequel we shall consider in particular the first uncountable cardinal in $\mathbf{L}$; it is denoted $\aleph_{1}^{\mathbf{L}}$. It is in fact an ordinal of $\mathbf{V}$ which is denoted $\omega_{1}^{\mathbf{L}}$. It is known that this ordinal satisfies the inequality $\omega_{1}^{\mathbf{L}} \leq \omega_{1}$. In a model $\mathbf{V}$ of the axiomatic system $\mathbf{Z F C}+\mathbf{V}=\mathbf{L}$ the equality $\omega_{1}^{\mathbf{L}}=\omega_{1}$ holds. But in some other models of ZFC the inequality may be strict and then $\omega_{1}^{\mathbf{L}}<\omega_{1}$. This is explained in [Jec02, page 202]: one can start from a model $\mathbf{V}$ of $\mathbf{Z F C}+\mathbf{V}=\mathbf{L}$ and construct by forcing a generic extension $\mathbf{V}[\mathbf{G}]$ in which the cardinals $\omega$ and $\omega_{1}$ are collapsed; in this extension the inequality $\omega_{1}^{\mathbf{L}}<\omega_{1}$ holds.

We now recall the notion of perfect set.

Definition 4.1. Let $P \subseteq \Sigma^{\omega}$, where $\Sigma$ is a finite alphabet having at least two letters. The set $P$ is said to be a perfect subset of $\Sigma^{\omega}$ if and only if :

(1) $P$ is a non-empty closed set, and 
(2) for every $x \in P$ and every open set $U$ containing $x$ there is an element $y \in P \cap U$ such that $x \neq y$.

So a perfect subset of $\Sigma^{\omega}$ is a non-empty closed set which has no isolated points. It is well known that a perfect subset of $\Sigma^{\omega}$ has cardinality $2^{\aleph_{0}}$, i.e. the cardinality of the continuum, see Mos80, page 66]. We recall now the definition of the perfect set property and some known results for Borel or analytic sets.

Definition 4.2. A class $\boldsymbol{\Gamma}$ of subsets of $\Sigma^{\omega}$ has the perfect set property iff each set $X \in \boldsymbol{\Gamma}$ is either countable or contains a perfect subset.

Theorem 4.3 (see [Mos80, Kec95]). The class of analytic subsets of $\Sigma^{\omega}$ has the perfect set property. In particular, the continuum hypothesis is satisfied for analytic sets: every analytic set is either countable or has cardinality $2^{\aleph_{0}}$.

On the other hand, "the perfect set property for the class of (effective) coanalytic subsets of $\Sigma^{\omega}$ " is actually independent from the axiomatic system ZFC. This fact follows easily, as we shall see below, from a result about the largest thin effective coanalytic set.

We first recall the notion of thin subset of $\Sigma^{\omega}$.

Definition 4.4. A set $X \subseteq \Sigma^{\omega}$ is said to be thin iff it contains no perfect subset.

The important following result was proved by Kechris Kec75] and independently by Guaspari Gua73] and Sacks [Sac76].

Theorem 4.5 (see [Mos80] page 247). Let $\Sigma$ be a finite alphabet having at least two letters. There exists a thin $\Pi_{1}^{1}$-set $\mathcal{C}_{1}\left(\Sigma^{\omega}\right) \subseteq \Sigma^{\omega}$ which contains every thin, $\Pi_{1}^{1}$-subset of $\Sigma^{\omega}$. It is called the largest thin $\Pi_{1}^{1}$-set in $\Sigma^{\omega}$.

Notice that the existence of the largest thin $\Pi_{1}^{1}$-set in $\Sigma^{\omega}$ is proved from the axiomatic system $\mathbf{Z F C}$, i.e. Zermelo-Fraenkel system $\mathbf{Z F}$ plus the axiom of choice $\mathbf{A C}$, and even if we replace the axiom of choice by a weaker version called the axiom of dependent choice $\mathbf{D C}$.

An important fact is that the cardinality of the largest thin $\Pi_{1}^{1}$-set in $\Sigma^{\omega}$ may depend on the model of ZFC.

We can now state Kechris's result on the cardinality of the largest thin $\Pi_{1}^{1}$-set, proved independently by Guaspari and Sacks, see also [Kan97, page 171].

Theorem 4.6. (ZFC) The cardinal of the largest thin $\Pi_{1}^{1}$-set in $\Sigma^{\omega}$ is equal to the cardinal of $\omega_{1}^{\mathbf{L}}$.

Notice that this means that in a given model $\mathbf{V}$ of $\mathbf{Z F C}$ the cardinal of the largest thin $\Pi_{1}^{1}$-set in $\Sigma^{\omega}$ is equal to the cardinal in $\mathbf{V}$ of the ordinal $\omega_{1}^{\mathbf{L}}$ which plays the role of the cardinal $\aleph_{1}$ in the inner model $\mathbf{L}$ of constructible sets of $\mathbf{V}$.

There exists also a largest thin $\Pi_{1}^{1}$-set in the Baire space $\omega^{\omega}$. By Mos80, Exercise 4F.7, page 251] the cardinal of the largest thin $\Pi_{1}^{1}$-set in the Baire space is equal to the cardinal of the largest thin $\Pi_{1}^{1}$-set in any Cantor space $\Sigma^{\omega}$ where $\Sigma$ is finite and has at least two elements.

We can now easily state the following result.

Corollary 4.7. The perfect set property for the class of effective coanalytic subsets of $\Sigma^{\omega}$ is independent from the axiomatic system $\mathbf{Z F C}$. Indeed it holds that :

(1) $(\mathbf{Z F C}+\mathbf{V}=\mathbf{L})$. The class of effective coanalytic subsets of $\Sigma^{\omega}$ does not have the perfect set property. 
(2) $\left(\mathbf{Z F C}+\omega_{1}^{\mathbf{L}}<\omega_{1}\right)$. The class of effective coanalytic subsets of $\Sigma^{\omega}$ has the perfect set property.

Proof.

(1) Assume first that $\mathbf{V}$ is a model of the axiomatic system $\mathbf{Z F C}+\mathbf{V}=\mathbf{L}$. In this model the cardinal of the largest thin $\Pi_{1}^{1}$-set in $\Sigma^{\omega}$ is equal to $\omega_{1}^{\mathbf{L}}=\omega_{1}$. Thus $\mathcal{C}_{1}\left(\Sigma^{\omega}\right)$ is not countable but it contains no perfect subset, hence the class of effective coanalytic subsets of $\Sigma^{\omega}$ does not have the perfect set property.

(2) Assume now that $\mathbf{V}$ is a model of the axiomatic system $\mathbf{Z F C}+\omega_{1}^{\mathbf{L}}<\omega_{1}$. In this model the largest thin $\Pi_{1}^{1}$-set in $\Sigma^{\omega}$ is countable. Thus every effective coanalytic subset of $\Sigma^{\omega}$ is either thin and countable or contains a perfect subset, hence the class of effective coanalytic subsets of $\Sigma^{\omega}$ has the perfect set property.

Notice that, by [Kan97, Theorem 14.10, page 184 and Theorem 11.6, page 136], the perfect set property for the class of all (boldface) $\boldsymbol{\Pi}_{1}^{1}$-subsets of $\Sigma^{\omega}$ is equiconsistent with the existence of an inaccessible cardinal, which is a large cardinal. The axiom "there exists an inaccessible cardinal" is a "large cardinal axiom"; its consistency can not be proved in ZFC. Thus the consistency of the perfect set property for the class of $\boldsymbol{\Pi}_{1}^{1}$-subsets of $\Sigma^{\omega}$ can not be proved in ZFC. We refer the reader to [Kan97] for an exposition of these results, which will not be necessary in this paper.

On the other hand, if in a model $\mathbf{V}$ of $\mathbf{Z F C}$ the class of $\boldsymbol{\Pi}_{1}^{1}$-subsets of $\Sigma^{\omega}$ fails to have the perfect property, we cannot infer from this property that the continuum hypothesis is satisfied for $\boldsymbol{\Pi}_{1}^{1}$-subsets of $\Sigma^{\omega}$. However every coanalytic set is the union of $\aleph_{1}$ Borel sets, and this implies that every coanalytic set is either countable, or of cardinality $\aleph_{1}$, or of cardinality $2^{\aleph_{0}}$, see JJec02, Corollary 25.16, page 488].

We can now state the following results which will be useful in the sequel.

Corollary 4.8. ( $\mathbf{Z F C}+\mathbf{V}=\mathbf{L})$ The largest thin $\Pi_{1}^{1}$-set in $\Sigma^{\omega}$ is not a Borel set.

Proof. In the model $\mathbf{L}$, the cardinal of the largest thin $\Pi_{1}^{1}$-set in $\Sigma^{\omega}$ is equal to the cardinal of $\omega_{1}^{\mathbf{L}}$. Moreover the continuum hypothesis is satisfied thus $2^{\aleph_{0}^{\mathbf{L}}}=\omega_{1}^{\mathbf{L}}$.

Thus the largest thin $\Pi_{1}^{1}$-set in $\Sigma^{\omega}$ has the cardinality of the continuum. But it has no perfect subset and the class of Borel sets has the perfect set property. Thus the largest thin $\Pi_{1}^{1}$-set in $\Sigma^{\omega}$ can not be a Borel set.

Corollary 4.9. (ZFC $\left.+\omega_{1}^{\mathbf{L}}<\omega_{1}\right)$ The largest thin $\Pi_{1}^{1}$-set in $\Sigma^{\omega}$ is countable, hence a $\Sigma_{2}^{0}$-set.

Proof. Let $\mathbf{V}$ be a model of $\left(\mathbf{Z F C}+\omega_{1}^{\mathbf{L}}<\omega_{1}\right)$. In this model $\omega_{1}$ is the first uncountable ordinal. Thus $\omega_{1}^{\mathbf{L}}<\omega_{1}$ implies that $\omega_{1}^{\mathbf{L}}$ is a countable ordinal in $\mathbf{V}$. Its cardinal is $\aleph_{0}$ and it is also the cardinal of the largest thin $\Pi_{1}^{1}$-set in $\Sigma^{\omega}$. Thus the set $\mathcal{C}_{1}\left(\Sigma^{\omega}\right)$ is countable. But for every $x \in \Sigma^{\omega}$ the singleton $\{x\}$ is a closed subset of $\Sigma^{\omega}$. Thus the largest thin $\Pi_{1}^{1}$-set in $\Sigma^{\omega}$ is a countable union of closed sets, i.e. a $\Sigma_{2}^{0}$-subset of $\Sigma^{\omega}$.

\section{Complexity of infinite COMputations}

There are several characterizations of the largest thin $\Pi_{1}^{1}$-set in $\Sigma^{\omega}$, see [Kec75, Mos80]. Moschovakis gave in [Mos80, page 248] a $\Pi_{1}^{1}$-formula $\phi$ defining the set $\mathcal{C}_{1}\left(\Sigma^{\omega}\right)$. Notice that all subformulas of this formula are themselves given previously in the book [Mos80].

From now on we shall simply denote by $\mathcal{C}_{1}$ the largest thin $\Pi_{1}^{1}$-set in $\{0,1\}^{\omega}=2^{\omega}$. 
This set $\mathcal{C}_{1}$ is a $\Pi_{1}^{1}$-set defined by a $\Pi_{1}^{1}$-formula $\phi$. Thus its complement $\mathcal{C}_{1}^{-}=2^{\omega}-\mathcal{C}_{1}$ is a $\Sigma_{1}^{1}$-set defined by the $\Sigma_{1}^{1}$-formula $\psi=\neg \phi$.

Recall that one can construct, from the $\Sigma_{1}^{1}$-formula $\psi$ defining $\mathcal{C}_{1}^{-}$, a Büchi Turing machine $\mathcal{T}$ accepting the $\omega$-language $\mathcal{C}_{1}^{-}$, see Sta97. We can then construct from the Büchi Turing machine $\mathcal{T}$, using a classical construction (see for instance [HMU01]), a 2-counter Büchi automaton $\mathcal{A}_{1}$ accepting the same $\omega$-language.

We are now going to recall some constructions which were used in a previous paper Fin06a to study topological properties of context-free $\omega$-languages, and which will be useful in the sequel.

Let $\Sigma=\{0,1\}, E$ be a new letter not in $\Sigma, S$ be an integer $\geq 1$, and $\theta_{S}: \Sigma^{\omega} \rightarrow$ $(\Sigma \cup\{E\})^{\omega}$ be the function defined, for all $x \in \Sigma^{\omega}$, by:

$$
\theta_{S}(x)=x(1) \cdot E^{S} \cdot x(2) \cdot E^{S^{2}} \cdot x(3) \cdot E^{S^{3}} \cdot x(4) \ldots x(n) \cdot E^{S^{n}} \cdot x(n+1) \cdot E^{S^{n+1}} \ldots
$$

We proved in Fin06a that if $L \subseteq \Sigma^{\omega}$ is an $\omega$-language in the class $\mathbf{B C L}(2)_{\omega}$ and $k=\operatorname{cardinal}(\Sigma)+2, S=(3 k)^{3}$, then one can construct effectively, from a Büchi 2-counter automaton $\mathcal{A}_{1}$ accepting $L$, a real time Büchi 8-counter automaton $\mathcal{A}_{2}$ such that $L\left(\mathcal{A}_{2}\right)=$ $\theta_{S}(L)$.

We used also in [Fin06a] another coding which we now recall. Let $K=2 \times 3 \times 5 \times 7 \times$ $11 \times 13 \times 17 \times 19=9699690$ be the product of the eight first prime numbers. Let $\Gamma$ be a finite alphabet; here we shall set $\Gamma=\Sigma \cup\{E\}$. An $\omega$-word $x \in \Gamma^{\omega}$ is coded by the $\omega$-word $h_{K}(x)=A \cdot C^{K} \cdot x(1) \cdot B \cdot C^{K^{2}} \cdot A \cdot C^{K^{2}} \cdot x(2) \cdot B \cdot C^{K^{3}} \cdot A \cdot C^{K^{3}} \cdot x(3) \cdot B \ldots B \cdot C^{K^{n}} \cdot A \cdot C^{K^{n}} \cdot x(n) \cdot B \ldots$ over the alphabet $\Gamma_{1}=\Gamma \cup\{A, B, C\}$, where $A, B, C$ are new letters not in $\Gamma$. In [Fin06a] we proved that, from a real time Büchi 8-counter automaton $\mathcal{A}_{2}$ accepting $L\left(\mathcal{A}_{2}\right) \subseteq \Gamma^{\omega}$, one can effectively construct a Büchi 1-counter automaton $\mathcal{A}_{3}$ accepting the $\omega$-language $h_{K}\left(L\left(\mathcal{A}_{2}\right)\right) \cup h_{K}\left(\Gamma^{\omega}\right)^{-}$.

Consider now the mapping $\phi_{K}:(\Gamma \cup\{A, B, C\})^{\omega} \rightarrow(\Gamma \cup\{A, B, C, F\})^{\omega}$ which is simply defined by: for all $x \in(\Gamma \cup\{A, B, C\})^{\omega}$,

$$
\phi_{K}(x)=F^{K-1} \cdot x(1) \cdot F^{K-1} \cdot x(2) \ldots F^{K-1} \cdot x(n) \cdot F^{K-1} \cdot x(n+1) \cdot F^{K-1} \ldots
$$

Then the $\omega$-language $\phi_{K}\left(L\left(\mathcal{A}_{3}\right)\right)=\phi_{K}\left(h_{K}\left(L\left(\mathcal{A}_{2}\right)\right) \cup h_{K}\left(\Gamma^{\omega}\right)^{-}\right)$is accepted by a real time Büchi 1-counter automaton $\mathcal{A}_{4}$ which can be effectively constructed from the real time Büchi 8-counter automaton $\mathcal{A}_{2}$, [Fin06a].

We can now use these previous constructions to obtain our first main result.

From now on we consider that we have obtained, from a Büchi Turing machine $\mathcal{T}$ accepting the $\omega$-language $\mathcal{C}_{1}^{-} \subseteq \Sigma^{\omega}=2^{\omega}$, a 2-counter Büchi automaton $\mathcal{A}_{1}$ accepting the same $\omega$-language, and then a real time Büchi 8-counter automaton $\mathcal{A}_{2}$ accepting the $\omega$-language $L\left(\mathcal{A}_{2}\right)=\theta_{S}\left(\mathcal{C}_{1}^{-}\right)$, where $S=(3 \times 4)^{3}=(12)^{3}$. Next, following the above construction, we have a Büchi 1-counter automaton $\mathcal{A}_{3}$ accepting the $\omega$-language $h_{K}\left(L\left(\mathcal{A}_{2}\right)\right) \cup h_{K}\left(\Gamma^{\omega}\right)^{-}$, and a real time Büchi 1-counter automaton $\mathcal{A}_{4}$ accepting the $\omega$-language $\phi_{K}\left(L\left(\mathcal{A}_{3}\right)\right)$. In the sequel we shall denote simply $\mathcal{A}_{4}$ by $\mathcal{A}$.

Theorem 5.1. Let $\mathcal{A}$ be the real-time 1-counter Büchi automaton constructed above. The topological complexity of the $\omega$-language $L(\mathcal{A})$ is not determined by the axiomatic system ZFC. Indeed it holds that:

(1) $(\mathbf{Z F C}+\mathbf{V}=\mathbf{L})$. The $\omega$-language $L(\mathcal{A})$ is a true analytic set.

(2) $\left(\mathbf{Z F C}+\omega_{1}^{\mathbf{L}}<\omega_{1}\right)$. The $\omega$-language $L(\mathcal{A})$ is a $\mathbf{\Pi}_{2}^{0}$-set. 
Proof.

(1) Assume first that $\mathbf{V}$ is a model of the axiomatic system $\mathbf{Z F C}+\mathbf{V}=\mathbf{L}$. In the model $\mathbf{V}$, by Corollary 4.8 the largest thin $\Pi_{1}^{1}$-set $\mathcal{C}_{1}$ is not a Borel set. Thus the $\omega$-language $\mathcal{C}_{1}^{-}=L\left(\mathcal{A}_{1}\right)$ is not a Borel set because the class of Borel subsets of $2^{\omega}$ is closed under complementation. The $\omega$-language $L\left(\mathcal{A}_{2}\right)=\theta_{S}\left(\mathcal{C}_{1}^{-}\right)$cannot be a Borel set. Indeed the function $\theta_{S}$ is continuous and if $L\left(\mathcal{A}_{2}\right)$ was Borel then the $\omega$-language $\mathcal{C}_{1}^{-}=\theta_{S}^{-1}\left(L\left(\mathcal{A}_{2}\right)\right)$ would be Borel too as the inverse image of a Borel set by a continuous function. Next we can see that the $\omega$-language $L\left(\mathcal{A}_{3}\right)=h_{K}\left(L\left(\mathcal{A}_{2}\right)\right) \cup h_{K}\left(\Gamma^{\omega}\right)^{-}$is not Borel. Indeed the function $h_{K}$ is also continuous and if $L\left(\mathcal{A}_{3}\right)$ was Borel then the $\omega$-language $L\left(\mathcal{A}_{2}\right)=$ $h_{K}^{-1}\left(L\left(\mathcal{A}_{3}\right)\right)$ would be Borel too as the inverse image of a Borel set by a continuous function. Finally we can see that the $\omega$-language $L(\mathcal{A})=\phi_{K}\left(L\left(\mathcal{A}_{3}\right)\right)$ is not Borel. Otherwise, the function $\phi_{K}$ being continuous, the $\omega$-language $L\left(\mathcal{A}_{3}\right)=\phi_{K}^{-1}(L(\mathcal{A}))$ would be Borel too as the inverse image of a Borel set by a continuous function. Thus the $\omega$-language $L(\mathcal{A})$ is an analytic but non Borel set.

(2) Assume now that $\mathbf{V}$ is a model of $\left(\mathbf{Z F C}+\omega_{1}^{\mathbf{L}}<\omega_{1}\right)$. In the model $\mathbf{V}$, by Corollary 4.9, the largest thin $\Pi_{1}^{1}$-set $\mathcal{C}_{1}$ is a $\boldsymbol{\Sigma}_{2}^{0}$-set. Thus its complement $\mathcal{C}_{1}^{-}=L\left(\mathcal{A}_{1}\right)$ is a $\boldsymbol{\Pi}_{2}^{0}$-set. It is then proved in Fin06a that the $\omega$-languages $L\left(\mathcal{A}_{2}\right)=\theta_{S}\left(\mathcal{C}_{1}^{-}\right), L\left(\mathcal{A}_{3}\right)=$ $h_{K}\left(L\left(\mathcal{A}_{2}\right)\right) \cup h_{K}\left(\Gamma^{\omega}\right)^{-}$, and finally $L(\mathcal{A})=\phi_{K}\left(L\left(\mathcal{A}_{3}\right)\right)$, are also $\boldsymbol{\Pi}_{2}^{0}$-sets.

We can now improve a recent result from [Fin09b. It is very natural to ask whether one can effectively determine the topological complexity of an $\omega$-language accepted by a given real-time 1-counter Büchi automaton (respectively, Büchi pushdown automaton). We had previously shown that this is not possible: For any countable ordinal $\alpha$, it is undecidable whether an $\omega$-language accepted by a given Büchi pushdown automaton is a $\Sigma_{\alpha}^{0}$-set (respectively, a $\boldsymbol{\Pi}_{\alpha}^{\mathbf{0}}$-set, a Borel set), Fin03. Moreover we have recently proved in Fin09b that these decision problems are actually $\Pi_{2}^{1}$-hard. Notice that here $\Pi_{2}^{1}$ is a class of the analytical hierarchy on subsets of $\mathbb{N}$. The notions of analytical hierarchy and of complete sets for classes of this hierarchy may be found for instance in the textbooks Rog67, Odi89, Odi99.

A real-time 1-counter Büchi automaton $\mathcal{C}$ has a finite description to which can be associated, in an effective way, a unique natural number called the index of $\mathcal{C}$. We have then a Gödel numbering of real-time 1-counter Büchi automata. From now on, we shall denote, as in Fin09b], $\mathcal{C}_{z}$ the real time Büchi 1-counter automaton of index $z$ (reading words over $\Omega=\{0,1, A, B, C, E, F\})$. The above cited result can be now formally stated as follows.

Theorem 5.2 (Fin09b). Let $\alpha$ be a countable ordinal. Then

(1) $\left\{z \in \mathbb{N} \mid L\left(\mathcal{C}_{z}\right)\right.$ is in the Borel class $\left.\boldsymbol{\Sigma}_{\alpha}^{0}\right\}$ is $\Pi_{2}^{1}$-hard.

(2) $\left\{z \in \mathbb{N} \mid L\left(\mathcal{C}_{z}\right)\right.$ is in the Borel class $\left.\Pi_{\alpha}^{0}\right\}$ is $\Pi_{2}^{1}$-hard.

(3) $\left\{z \in \mathbb{N} \mid L\left(\mathcal{C}_{z}\right)\right.$ is a Borel set $\}$ is $\Pi_{2}^{1}$-hard.

This implies in particular that these decison problems are not in the class $\Sigma_{2}^{1}$, but they still could have been $\Pi_{2}^{1}$-complete. We are going now to prove that this is not the case.

Theorem 5.3. Let $\alpha$ be a countable ordinal. Then

(1) For $\alpha>2,\left\{z \in \mathbb{N} \mid L\left(\mathcal{C}_{z}\right)\right.$ is in the Borel class $\left.\boldsymbol{\Sigma}_{\alpha}^{0}\right\}$ is not a $\Pi_{2}^{1}$-set.

(2) For $\alpha \geq 2,\left\{z \in \mathbb{N} \mid L\left(\mathcal{C}_{z}\right)\right.$ is in the Borel class $\left.\Pi_{\alpha}^{0}\right\}$ is not a $\Pi_{2}^{1}$-set.

(3) $\left\{z \in \mathbb{N} \mid L\left(\mathcal{C}_{z}\right)\right.$ is a Borel set $\}$ is not a $\Pi_{2}^{1}$-set. 
Proof. We first prove item (1). Let $\mathcal{A}$ be the real-time 1-counter Büchi automaton cited in Theorem 5.1 and let $z_{0}$ be its index so that $\mathcal{A}=\mathcal{C}_{z_{0}}$.

Assume now that $\mathbf{V}$ is a model of $\left(\mathbf{Z F C}+\omega_{1}^{\mathbf{L}}<\omega_{1}\right)$. In the model $\mathbf{V}$, by Theorem 5.1, the $\omega$-language $L(\mathcal{A})$ is a $\boldsymbol{\Pi}_{2}^{0}$-set, hence also a $\boldsymbol{\Sigma}_{\alpha}^{0}$-set for any countable ordinal $\alpha>2$. Thus, for $\alpha>2$, the integer $z_{0}$ belongs to the set $\left\{z \in \mathbb{N} \mid L\left(\mathcal{C}_{z}\right)\right.$ is in the Borel class $\left.\boldsymbol{\Sigma}_{\alpha}^{0}\right\}$.

But, by Theorem [5.1, in the inner model $\mathbf{L} \subseteq \mathbf{V}$, the $\omega$-language $L(\mathcal{A})$ is an analytic but non Borel set so the integer $z_{0}$ does not belong to the set $\left\{z \in \mathbb{N} \mid L\left(\mathcal{C}_{z}\right)\right.$ is in the Borel class $\left.\boldsymbol{\Sigma}_{\alpha}^{0}\right\}$.

On the other hand, Shoenfield's Absoluteness Theorem implies that every $\Sigma_{2}^{1}$-set (respectively, $\Pi_{2}^{1}$-set) is absolute for all inner models of ( $\left.\mathrm{ZF}+\mathrm{DC}\right)$, where (DC) is the weak version of the axiom of choice called the axiom of dependent choice which holds in particular in the inner model $\mathbf{L}$, see [Jec02, page 490].

In particular, if the set $\left\{z \in \mathbb{N} \mid L\left(\mathcal{C}_{z}\right)\right.$ is in the Borel class $\left.\boldsymbol{\Sigma}_{\alpha}^{0}\right\}$ was a $\Pi_{2}^{1}$-set, then it could not be a different subset of $\mathbb{N}$ in the models $\mathbf{V}$ and $\mathbf{L}$ considered above. Therefore, for any countable ordinal $\alpha>2$, the set $\left\{z \in \mathbb{N} \mid L\left(\mathcal{C}_{z}\right)\right.$ is in the Borel class $\left.\boldsymbol{\Sigma}_{\alpha}^{0}\right\}$ is not a $\Pi_{2}^{1}$-set.

Items (2) and (3) follow similarly from Theorem 5.1 and from Shoenfield's Absoluteness Theorem.

In order to prove similar results for infinitary rational relations accepted by 2-tape Büchi automata, we shall use a construction from [Fin06b]. We proved in [Fin06b] that infinitary rational relations have the same topological complexity as $\omega$-languages accepted by Büchi Turing machines. We used a simulation of the behaviour of real time 1-counter automata by 2 -tape Büchi automata. We recall now a coding which was used in [Fin06b].

We first define a coding of an $\omega$-word over the finite alphabet $\Omega=\{0,1, A, B, C, E, F\}$ by an $\omega$-word over the alphabet $\Omega^{\prime}=\Omega \cup\{D\}$, where $D$ is an additional letter not in $\Omega$. For $x \in \Omega^{\omega}$ the $\omega$-word $h(x)$ is defined by :

$$
h(x)=D \cdot 0 \cdot x(1) \cdot D \cdot 0^{2} \cdot x(2) \cdot D \cdot 0^{3} \cdot x(3) \cdot D \ldots D \cdot 0^{n} \cdot x(n) \cdot D \cdot 0^{n+1} \cdot x(n+1) \cdot D \ldots
$$

It is easy to see that the mapping $h$ from $\Omega^{\omega}$ into $(\Omega \cup\{D\})^{\omega}$ is continuous and injective.

Let now $\alpha$ be the $\omega$-word over the alphabet $\Omega^{\prime}$ which is simply defined by:

$$
\alpha=D \cdot 0 \cdot D \cdot 0^{2} \cdot D \cdot 0^{3} \cdot D \cdot 0^{4} \cdot D \ldots D \cdot 0^{n} \cdot D \cdot 0^{n+1} \cdot D \ldots
$$

The following results were proved in Fin06b.

Lemma 5.4 ([Fin06b]). Let $\Omega$ be a finite alphabet such that $0 \in \Omega, \alpha$ be the $\omega$-word over $\Omega \cup\{D\}$ defined as above, and $L \subseteq \Omega^{\omega}$ be in $\mathbf{r}-\mathbf{B C L}(1)_{\omega}$. Then there exists an infinitary rational relation $R_{1} \subseteq(\Omega \cup\{D\})^{\omega} \times(\Omega \cup\{D\})^{\omega}$ such that:

$$
\forall x \in \Omega^{\omega}(x \in L) \text { iff }\left((h(x), \alpha) \in R_{1}\right)
$$

Lemma 5.5 (Fin06b]). The set $R_{2}=(\Omega \cup\{D\})^{\omega} \times(\Omega \cup\{D\})^{\omega}-\left(h\left(\Omega^{\omega}\right) \times\{\alpha\}\right)$ is an infinitary rational relation.

Considering the union $R_{1} \cup R_{2}$ of the two infinitary rational relations obtained in the two above lemmas we get the following result.

Proposition 5.6 (Fin06b] $)$. Let $L \subseteq \Omega^{\omega}$ be in $\mathbf{r - B C L}(1)_{\omega}$ and $\mathcal{L}=h(L) \cup\left(h\left(\Omega^{\omega}\right)\right)^{-}$. Then

$$
R=\mathcal{L} \times\{\alpha\} \bigcup\left(\Omega^{\prime}\right)^{\omega} \times\left(\left(\Omega^{\prime}\right)^{\omega}-\{\alpha\}\right)
$$


is an infinitary rational relation. Moreover one can construct effectively, from a real time 1-counter Büchi automaton $\mathcal{A}$ accepting L, a 2-tape Büchi automaton $\mathcal{B}$ accepting the infinitary relation $R$.

Let now $\mathcal{A}$ be the real time 1-counter Büchi automaton constructed above and cited in Theorem 5.1 and $\mathcal{B}$ be the 2-tape Büchi automaton which can be constructed from $\mathcal{A}$ by the above Proposition 5.6. We can now state our second main result.

Theorem 5.7. The topological complexity of the infinitary rational relation $L(\mathcal{B})$ is not determined by the axiomatic system ZFC. Indeed it holds that :

(1) $(\mathbf{Z F C}+\mathbf{V}=\mathbf{L})$. The relation $L(\mathcal{B})$ is a true analytic set.

(2) $\left(\mathbf{Z F C}+\omega_{1}^{\mathbf{L}}<\omega_{1}\right)$. The relation $L(\mathcal{B})$ is a $\mathbf{\Pi}_{2}^{0}$-set.

Proof.

(1) Assume first that $\mathbf{V}$ is a model of the axiomatic system $\mathbf{Z F C}+\mathbf{V}=\mathbf{L}$. In the model $\mathbf{V}$, by Corollary 4.8 the largest thin $\Pi_{1}^{1}$-set $\mathcal{C}_{1}$ is not a Borel set and by Theorem 5.1 the $\omega$-language $L(\mathcal{A})$ is a true analytic set.

On the other hand the function $h$ is continuous. Thus the function $g$ from $\Omega^{\omega}$ into $(\Omega \cup\{D\})^{\omega} \times(\Omega \cup\{D\})^{\omega}$ defined by $g(x)=(h(x), \alpha)$ is also continuous. If the relation $L(\mathcal{B})$ was a Borel set then the $\omega$-language $L(\mathcal{A})=g^{-1}(L(\mathcal{B}))$ would be also a Borel set as the inverse image of a Borel set by a continuous function. Thus the relation $L(\mathcal{B})$ is not a Borel set.

(2) Assume now that $\mathbf{V}$ is a model of $\left(\mathbf{Z F C}+\omega_{1}^{\mathbf{L}}<\omega_{1}\right)$. In the model $\mathbf{V}$, by Corollary 4.9, the largest thin $\Pi_{1}^{1}$-set $\mathcal{C}_{1}$ is a $\boldsymbol{\Sigma}_{2}^{0}$-set and by Theorem 5.1 the $\omega$-language $L(\mathcal{A})$ is a $\Pi_{2}^{0}$-set. It is easy to prove that $\mathcal{L}=h(L(\mathcal{A})) \cup\left(h\left(\Omega^{\omega}\right)\right)^{-}$is also a $\boldsymbol{\Pi}_{2}^{0}$-set (this is due to the fact that $h$ is an homeomorphism between $\Omega^{\omega}$ and its image $h\left(\Omega^{\omega}\right)$ which is a closed subset of $(\Omega \cup\{D\})^{\omega}$, see Fin06b). Then one can easily see that the set $\mathcal{L} \times\{\alpha\}$ is also a $\Pi_{2}^{0}$-set. But the set $\left(\Omega^{\prime}\right)^{\omega} \times\left(\left(\Omega^{\prime}\right)^{\omega}-\{\alpha\}\right)$ is an open hence $\boldsymbol{\Pi}_{2}^{0}$-subset of $(\Omega \cup\{D\})^{\omega} \times(\Omega \cup\{D\})^{\omega}$. Thus the relation $R=\mathcal{L} \times\{\alpha\} \cup\left(\Omega^{\prime}\right)^{\omega} \times\left(\left(\Omega^{\prime}\right)^{\omega}-\{\alpha\}\right)$ is a $\Pi_{2}^{0}$-subset of $(\Omega \cup\{D\})^{\omega} \times(\Omega \cup\{D\})^{\omega}$.

From now on we shall denote $\mathcal{T}_{z}$ the 2 -tape Büchi automaton of index $z$. Then we recall the following recent result which shows that topological properties of infinitary rational relations are highly undecidable.

Theorem 5.8 ( [Fin09b] $)$. Let $\alpha$ be a non null countable ordinal. Then

(1) $\left\{z \in \mathbb{N} \mid L\left(\mathcal{T}_{z}\right)\right.$ is in the Borel class $\left.\boldsymbol{\Sigma}_{\alpha}^{0}\right\}$ is $\Pi_{2}^{1}$-hard.

(2) $\left\{z \in \mathbb{N} \mid L\left(\mathcal{T}_{z}\right)\right.$ is in the Borel class $\left.\Pi_{\alpha}^{0}\right\}$ is $\Pi_{2}^{1}$-hard.

(3) $\left\{z \in \mathbb{N} \mid L\left(\mathcal{T}_{z}\right)\right.$ is a Borel set $\}$ is $\Pi_{2}^{1}$-hard.

We can now state that these decision problems are not in the class $\Pi_{2}^{1}$.

Theorem 5.9. Let $\alpha$ be a countable ordinal. Then

(1) For $\alpha>2,\left\{z \in \mathbb{N} \mid L\left(\mathcal{T}_{z}\right)\right.$ is in the Borel class $\left.\Sigma_{\alpha}^{0}\right\}$ is not a $\Pi_{2}^{1}$-set.

(2) For $\alpha \geq 2,\left\{z \in \mathbb{N} \mid L\left(\mathcal{T}_{z}\right)\right.$ is in the Borel class $\left.\Pi_{\alpha}^{0}\right\}$ is not a $\Pi_{2}^{1}$-set.

(3) $\left\{z \in \mathbb{N} \mid L\left(\mathcal{T}_{z}\right)\right.$ is a Borel set $\}$ is not a $\Pi_{2}^{1}$-set.

Proof. We can reason as in the proof of Theorem 5.3 (in the case of $\omega$-languages of 1-counter Büchi automata). We use Shoenfield's Absoluteness Theorem and Theorem 5.7 instead of Theorem 5.1, 
We consider now Büchi recognizable languages of infinite pictures. We shall use in the sequel a result proved in [Fin04, Fin09a] which we now recall.

For $\sigma \in \Sigma^{\omega}=\{0,1\}^{\omega}$ we denote $\sigma^{0}$ the $\omega$-picture whose first row is the $\omega$-word $\sigma$ and whose other rows are labelled with the letter 0 . For an $\omega$-language $L \subseteq \Sigma^{\omega}=\{0,1\}^{\omega}$ we denote $L^{0}$ the language of infinite pictures $\left\{\sigma^{0} \mid \sigma \in L\right\}$.

Lemma 5.10 ([Fin04]). If $L \subseteq \Sigma^{\omega}$ is accepted by some Turing machine with a Büchi acceptance condition, then $L^{0}$ is Büchi recognizable by a finite tiling system.

Recall that for $\Gamma$ a finite alphabet having at least two letters, the set $\Gamma^{\omega \times \omega}$ of functions from $\omega \times \omega$ into $\Gamma$ is usually equipped with the product topology of the discrete topology on $\Gamma$. This topology may be defined by the following distance $d$. Let $x$ and $y$ in $\Gamma^{\omega \times \omega}$ such that $x \neq y$, then

$$
d(x, y)=\frac{1}{2^{n}}, \quad \text { where } \quad n=\min \{p \geq 0 \mid \exists(i, j) x(i, j) \neq y(i, j) \text { and } i+j=p\} .
$$

Then the topological space $\Gamma^{\omega \times \omega}$ is homeomorphic to the topological space $\Gamma^{\omega}$, equipped with the Cantor topology.

The set $\Sigma^{\omega, \omega}$ of $\omega$-pictures over $\Sigma$, viewed as a topological subspace of $\hat{\Sigma}^{\omega \times \omega}$, is easily seen to be homeomorphic to the topological space $\Sigma^{\omega \times \omega}$, via the mapping $\varphi: \Sigma^{\omega, \omega} \rightarrow \Sigma^{\omega \times \omega}$ defined by $\varphi(p)(i, j)=p(i+1, j+1)$ for all $p \in \Sigma^{\omega, \omega}$ and $i, j \in \omega$.

Let now $\mathcal{T}$ be a Büchi Turing machine accepting the $\omega$-language $\mathcal{C}_{1}^{-}$. Using Lemma 5.10 we can construct a Büchi tiling system $\mathcal{S}$ accepting the $\omega$-picture language $\left(\mathcal{C}_{1}^{-}\right)^{0}$. We consider now the topological complexity of this set $L(\mathcal{S}) \subseteq \Sigma^{\omega, \omega}$

It is then easy to see that if $L \subseteq \Sigma^{\omega}=\{0,1\}^{\omega}$ is a $\Pi_{2}^{0}$-subset of $\Sigma^{\omega}$ then the $\omega$-picture language $L^{0}$ is a $\Pi_{2}^{0}$-subset of $\Sigma^{\omega, \omega}$. And if $L \subseteq \Sigma^{\omega}=\{0,1\}^{\omega}$ is not Borel then the $\omega$-picture language $L^{0}$ is also not Borel. Then Corollaries 4.8 and 4.9 imply the following result.

Theorem 5.11. The topological complexity of the $\omega$-picture language $L(\mathcal{S})$ is not determined by the axiomatic system ZFC. Indeed it holds that :

(1) $(\mathbf{Z F C}+\mathbf{V}=\mathbf{L})$. The $\omega$-picture language $L(\mathcal{S})$ is a true analytic set.

(2) $\left(\mathbf{Z F C}+\omega_{1}^{\mathbf{L}}<\omega_{1}\right)$. The $\omega$-picture language $L(\mathcal{S})$ is a $\mathbf{\Pi}_{2}^{0}$-set.

We have recently proved that the topological complexity of $\omega$-picture languages accepted by Büchi tiling systems is highly undecidable. Below the Büchi tiling system of index $z$ is denoted by $\mathcal{S}_{z}$.

Theorem 5.12 ( [Fin09a $]$ ). Let $\alpha$ be a non null countable ordinal. Then

(1) $\left\{z \in \mathbb{N} \mid L\left(\mathcal{S}_{z}\right)\right.$ is in the Borel class $\left.\boldsymbol{\Sigma}_{\alpha}^{0}\right\}$ is $\Pi_{2}^{1}$-hard.

(2) $\left\{z \in \mathbb{N} \mid L\left(\mathcal{S}_{z}\right)\right.$ is in the Borel class $\left.\Pi_{\alpha}^{0}\right\}$ is $\Pi_{2}^{1}$-hard.

(3) $\left\{z \in \mathbb{N} \mid L\left(\mathcal{S}_{z}\right)\right.$ is a Borel set $\}$ is $\Pi_{2}^{1}$-hard.

As in the case of $\omega$-languages of 1-counter automata or of 2-tape automata, we can now infer the following result from Shoenfield's Absoluteness Theorem and Theorem 5.11 .

Theorem 5.13. Let $\alpha$ be a countable ordinal. Then

(1) For $\alpha>2,\left\{z \in \mathbb{N} \mid L\left(\mathcal{S}_{z}\right)\right.$ is in the Borel class $\left.\boldsymbol{\Sigma}_{\alpha}^{0}\right\}$ is not a $\Pi_{2}^{1}$-set.

(2) For $\alpha \geq 2,\left\{z \in \mathbb{N} \mid L\left(\mathcal{S}_{z}\right)\right.$ is in the Borel class $\left.\Pi_{\alpha}^{0}\right\}$ is not a $\Pi_{2}^{1}$-set.

(3) $\left\{z \in \mathbb{N} \mid L\left(\mathcal{S}_{z}\right)\right.$ is a Borel set $\}$ is not a $\Pi_{2}^{1}$-set. 


\section{Concluding Remarks}

We obtained surprising results which show that the topological complexity of an $\omega$ language accepted by a 1-counter Büchi automaton, of an infinitary rational relation accepted by a 2-tape Büchi automaton, or of a Büchi recognizable language of infinite pictures, is not determined by the axiomatic system ZFC.

We have inferred from the proof of the above results and from Shoenfield's Absoluteness Theorem an improvement of the lower bound of some decision problems recently studied in [Fin09b, Fin09a].

Recall that, by [Fin09b, Remark 3.25], if $\alpha$ is an ordinal smaller than the Church-Kleene ordinal $\omega_{1}^{\mathrm{CK}}$, which is the first non-recursive ordinal, then $\left\{z \in \mathbb{N} \mid L\left(\mathcal{C}_{z}\right)\right.$ is in the Borel class $\left.\boldsymbol{\Sigma}_{\alpha}^{0}\right\}$ (respectively, $\left\{z \in \mathbb{N} \mid L\left(\mathcal{C}_{z}\right)\right.$ is in the Borel class $\left.\boldsymbol{\Pi}_{\alpha}^{0}\right\}$ ) is a $\Sigma_{3}^{1}$-set. We now know that for $\alpha>2$ (respectively, $\alpha \geq 2$ ), it is actually in the class $\Sigma_{3}^{1} \backslash\left(\Sigma_{2}^{1} \cup \Pi_{2}^{1}\right)$ but the question is still open whether these problems are $\Sigma_{3}^{1}$-complete. The exact complexity of being in the Borel class $\boldsymbol{\Sigma}_{\alpha}^{0}$ (respectively, $\boldsymbol{\Pi}_{\alpha}^{0}$ ), for a countable ordinal $\alpha$, remains an open problem for $\omega$-languages of real time 1-counter automata (respectively, pushdown automata, 2-tape automata) and for Büchi recognizable languages of infinite pictures.

\section{ACKNOWLEDGEMENT}

I wish to thank the referees for their very useful comments on a preliminary version of this paper.

\section{REFERENCES}

[ABB96] J.-M. Autebert, J. Berstel, and L. Boasson. Context free languages and pushdown automata. In Handbook of formal languages, Vol. 1. Springer-Verlag, 1996.

[ATW03] J.-H. Altenbernd, W. Thomas, and S. Wöhrle. Tiling systems over infinite pictures and their acceptance conditions. In Proceedings of the 6th International Conference Developments in Language Theory, DLT 2002, volume 2450 of Lecture Notes in Computer Science, pages 297-306. Springer, 2003.

[BJ08] A. Ballier and E. Jeandel. Tilings and model theory. In Proceedings of the Journées Automates Cellulaires 2008, Uzès, France, pages 29-39, 2008.

[Büc62] J.R. Büchi. On a decision method in restricted second order arithmetic. In Stanford University Press, editor, Proceedings of the 1960 International Congress on Logic Methodology and Philosophy of Science, pages 1-11. Stanford University Press, 1962.

[CG78] R.S. Cohen and A.Y. Gold. $\omega$-computations on Turing machines. Theoretical Computer Science, 6:1-23, 1978.

[EH93] J Engelfriet and H. J. Hoogeboom. X-automata on $\omega$-words. Theoretical Computer Science, 110(1):1-51, 1993.

[Fin01] O. Finkel. Topological properties of omega context free languages. Theoretical Computer Science, 262(1-2):669-697, 2001.

[Fin03] O. Finkel. Borel hierarchy and omega context free languages. Theoretical Computer Science, 290(3):1385-1405, 2003.

[Fin04] O. Finkel. On recognizable languages of infinite pictures. International Journal of Foundations of Computer Science, 15(6):823-840, 2004.

[Fin06a] O. Finkel. Borel ranks and Wadge degrees of omega context free languages. Mathematical Structures in Computer Science, 16(5):813-840, 2006.

[Fin06b] O. Finkel. On the accepting power of two-tape Büchi automata. In Proceedings of the 23rd International Symposium on Theoretical Aspects of Computer Science, STACS 2006, volume 3884 of Lecture Notes in Computer Science, pages 301-312. Springer, 2006. 
[Fin08a] O. Finkel. Topological complexity of context free $\omega$-languages: A survey. 2008. preprint, available from http://fr.arxiv.org/abs/0806.1413.

[Fin08b] O. Finkel. Wadge degrees of infinitary rational relations. Special Issue on Intensional Programming and Semantics in honour of Bill Wadge on the occasion of his 60th cycle, Mathematics in Computer Science, 2(1):85-102, 2008.

[Fin09a] O. Finkel. Highly undecidable problems about recognizability by tiling systems. Fundamenta Informaticae, 91(2):305-323, 2009.

[Fin09b] O. Finkel. Highly undecidable problems for infinite computations. RAIRO-Theoretical Informatics and Applications, 43(2):339-364, 2009.

[Gir81] F. Gire. Relations rationnelles infinitaires. PhD thesis, Université Paris VII, 1981.

[GN84] F. Gire and M. Nivat. Relations rationnelles infinitaires. Calcolo, pages 91-125, 1984.

[Gua73] D. Guaspari. Thin and well ordered analytical sets. PhD thesis, Cambridge University, 1973.

[HMU01] J. E. Hopcroft, R. Motwani, and J. D. Ullman. Introduction to automata theory, languages, and computation. Addison-Wesley Publishing Co., Reading, Mass., 2001. Addison-Wesley Series in Computer Science.

[Jec02] T. Jech. Set theory, third edition. Springer, 2002.

[Kan97] A. Kanamori. The Higher Infinite. Springer-Verlag, 1997.

[Kec75] A.S. Kechris. The theory of countable analytical sets. Transactions of the American Mathematical Society, 202:259-297, 1975.

[Kec95] A. S. Kechris. Classical descriptive set theory. Springer-Verlag, New York, 1995.

[Lan69] L.H. Landweber. Decision problems for w-automata. Mathematical Systems Theory, 3(4):376-384, 1969.

[LT94] H. Lescow and W. Thomas. Logical specifications of infinite computations. In J. W. de Bakker, Willem P. de Roever, and Grzegorz Rozenberg, editors, A Decade of Concurrency, volume 803 of Lecture Notes in Computer Science, pages 583-621. Springer, 1994.

[Mos80] Y. N. Moschovakis. Descriptive set theory. North-Holland Publishing Co., Amsterdam, 1980.

[Odi89] P.G. Odifreddi. Classical Recursion Theory, Vol I, volume 125 of Studies in Logic and the Foundations of Mathematics. North-Holland Publishing Co., Amsterdam, 1989.

[Odi99] P.G. Odifreddi. Classical Recursion Theory, Vol II, volume 143 of Studies in Logic and the Foundations of Mathematics. North-Holland Publishing Co., Amsterdam, 1999.

[PP04] D. Perrin and J.-E. Pin. Infinite words, automata, semigroups, logic and games, volume 141 of Pure and Applied Mathematics. Elsevier, 2004.

[Rog67] H. Rogers. Theory of Recursive Functions and Effective Computability. McGraw-Hill, New York, 1967.

[Sac76] G.E. Sacks. Countable admissible ordinals and hyperdegrees. Advances in Mathematics, 171:213262, 1976.

[Sim92] P. Simonnet. Automates et théorie descriptive. PhD thesis, Université Paris VII, 1992.

[Sta86] L. Staiger. Hierarchies of recursive $\omega$-languages. Elektronische Informationsverarbeitung und Kybernetik, 22(5-6):219-241, 1986.

[Sta87] L. Staiger. Research in the theory of $\omega$-languages. Journal of Information Processing and Cybernetics, 23(8-9):415-439, 1987.

[Sta97] L. Staiger. w-languages. In Handbook of formal languages, Vol. 3, pages 339-387. Springer, Berlin, 1997.

[Tho90] W. Thomas. Automata on infinite objects. In J. van Leeuwen, editor, Handbook of Theoretical Computer Science, volume B, Formal models and semantics, pages 135-191. Elsevier, 1990. 